Article

\title{
Unraveling the Peculiarities in the Temperature-Dependent Structural Evolution of Black Phosphorus
}

\author{
Boby Joseph ${ }^{1, *}$, Nicola Demitri ${ }^{1}$, Paolo Lotti ${ }^{1}$, Andrea Lausi ${ }^{1}$ and Paolo Dore ${ }^{2}$ \\ 1 Elettra-Sincrotrone Trieste, Area Science Park, 34149 Basovizza, Italy; nicola.demitri@elettra.eu (N.D.); \\ paolo.lotti@elettra.eu (P.L.); andrea.lausi@elettra.eu (A.L.) \\ 2 Department of Physics, Sapienza University of Rome, Piazzale Aldo Moro 2, 00185 Rome, Italy; \\ paolo.dore@roma1.infn.it \\ * Correspondence: boby.joseph@gmail.com; Tel.: +39-366-6907300
}

Academic Editors: Augusto Marcelli and Antonio Bianconi Received: 9 January 2017; Accepted: 16 February 2017; Published: 20 February 2017

\begin{abstract}
Black phosphorous (BP) is one of the important emerging two-dimensional systems. We have undertaken a structural investigation of $\mathrm{BP}$ in the temperature range of $320 \mathrm{~K}$ to $85 \mathrm{~K}$ using synchrotron X-ray diffraction (XRD) studies. The XRD pattern of BP is heavily influenced by the preferred orientation effects. Collection of the diffraction pattern in a standard capillary geometry with controlled capillary rotations perpendicular to the X-ray direction permitted us to provide insights to the effects of the preferred orientation. In the range of $320 \mathrm{~K}$ to $85 \mathrm{~K}$, BP remains in the so-called "A17" orthorhombic structure. Lattice parameters show a regular shrinkage with the lowering of the temperature as expected for any elemental metallic system. Dense temperature sampling permitted us to observe a small but clear deviation from the linear behavior in of one of the in-plane lattice parameters. This temperature-dependent structural evolution seems to provide some insights into the temperature dependence of the macroscopic properties of BP such as the Hall coefficient, thermal conductivity, etc.
\end{abstract}

Keywords: two-dimensional systems; black phosphorus; $\mathrm{x}$-ray powder diffraction; transport properties

\section{Introduction}

Understanding the structure-function relationship is an important guiding force for research across different branches of science. In solid-state physics, this is indeed a strong driving force behind the intense investigations of novel materials. Human development is critically dependent on improvement in the functionalities of materials. In this context, development of new materials or modifying existing materials for newer applications are important milestones in human development. Every newly discovered material is always intensely investigated both to improve the properties and to correlate the structural properties with its functional behaviors. To cite some examples from a large volume of the research work already accumulated, we can consider the case of the high-temperature cuprate superconductors. Recently, in this class of materials, it was found that oxygen ordering plays a critical role in determining the macroscopic properties of the system [1-3]. In fact, our own research works in diverse materials such as hydrogen storage materials [4], lithium battery materials [5], several nano-material systems [6-8], newly discovered Fe-based superconductors [9-12], novel magnetic materials $[13,14]$ and solar cell materials [15], etc., were indeed aimed at understanding the structure-function relations. Black phosphorus (BP) is an emerging two-dimensional system offering several perspectives for applications. Complementarities of this system with graphene and its potential technological applications are of high current interest. Except for citing three recent 
reviews [16-18], we do not dwell further into such details. Here, we explore the structural behavior of $\mathrm{BP}$ as a function of temperature from $320 \mathrm{~K}$ to $85 \mathrm{~K}$. The results show a differing temperature dependence of the three crystal lattice parameters. The temperature-dependent results shed light on temperature-dependent macroscopic properties such as the thermal conductivity, Hall coefficient, etc.

\section{Results}

We organized the results of the present investigation into three subsections. Ambient condition X-ray diffraction data is described in Section 2.1; structural analysis of the ambient temperature data is presented in Section 2.2; and temperature-dependent structural properties are described and are correlated with macroscopic properties of BP in Section 2.3.

\subsection{Preferred Orientation Effects in Black Phosphorus XRD Spectrum}

Black phosphorus, prepared first time by Bridgeman in 1914 [19], has an orthorhombic A17 structure [20]. A structural model is given in the inset of Figure 1. The unit-cell contains eight atoms with the atoms arranged in puckered layers. Intra-layer bonding is of the covalent type, with each phosphorus atom surrounded by three neighboring phosphorus atoms at the same distance, while different layers are held by van der Waals forces. This structural arrangement gives an easy exfoliation possibility for the two-dimensional layers, which recently has significantly increased the interest in this system [16,17]. This effect manifests while powdering the bulk sample using a mortar and pestle. Similar to the graphite case, rather than breaking into microcrystallites, layers peel off from the bulk system. Loading such a system into a capillary tube or a diamond anvil cell for high pressure can provide a diffraction pattern with different peaks having intensities very different from what may be expected from an isotropic powder of the system. This can result in varying intensities of diffraction peaks for a given acquisition time by varying the amount of capillary rotation angles perpendicular to the X-ray beam during the diffraction data collection. Figure 1 clearly demonstrates such an effect where a fixed collection time of $10 \mathrm{~s}$ with varying rotation angles results in a large variation in the intensities of some of the peaks. In particular, from Figure 1 we can note a large variation in the intensity of the (020) peak (as expected, there is similar behavior also for the (040) peak), whereas a much weaker effect is seen at the (021) peak. BP layers are bound along the out-of-plane direction (lattice direction $b$ ) via the van der Waals interaction, leaving relatively larger interlayer separation. However, in the plane, the P-P atoms are more strongly bound due to covalent interactions. There are two distinct atomic arrangements, viz. armchair and zigzag configurations, in the two perpendicular directions, as can be readily appreciated from the inset of Figure 2. Obviously, such a distinct atomic arrangement is supposed to provide some kind of distinct response to external conditions such as pressure and temperature, and thus the corresponding macroscopic properties.

In Figure 2 we present the X-ray diffraction data of BP collected using a capillary with photons of the wavelength $0.5007 \AA$ in spinning and without spinning configurations. Obviously, due to a better average, the spinning of the capillary helps in minimizing the preferred orientation effects. We remark that such a spinning configuration is not possible in several experimental configurations, for example in high-pressure measurements using a diamond anvil cell. The preferred orientation effects can be easily incorporated in several modern diffraction refinement packages. Indeed, for the analysis of the diffraction data of two-dimensional systems, a standard capillary geometry is also required for the inclusion of preferred orientation effects, as in the present case. 


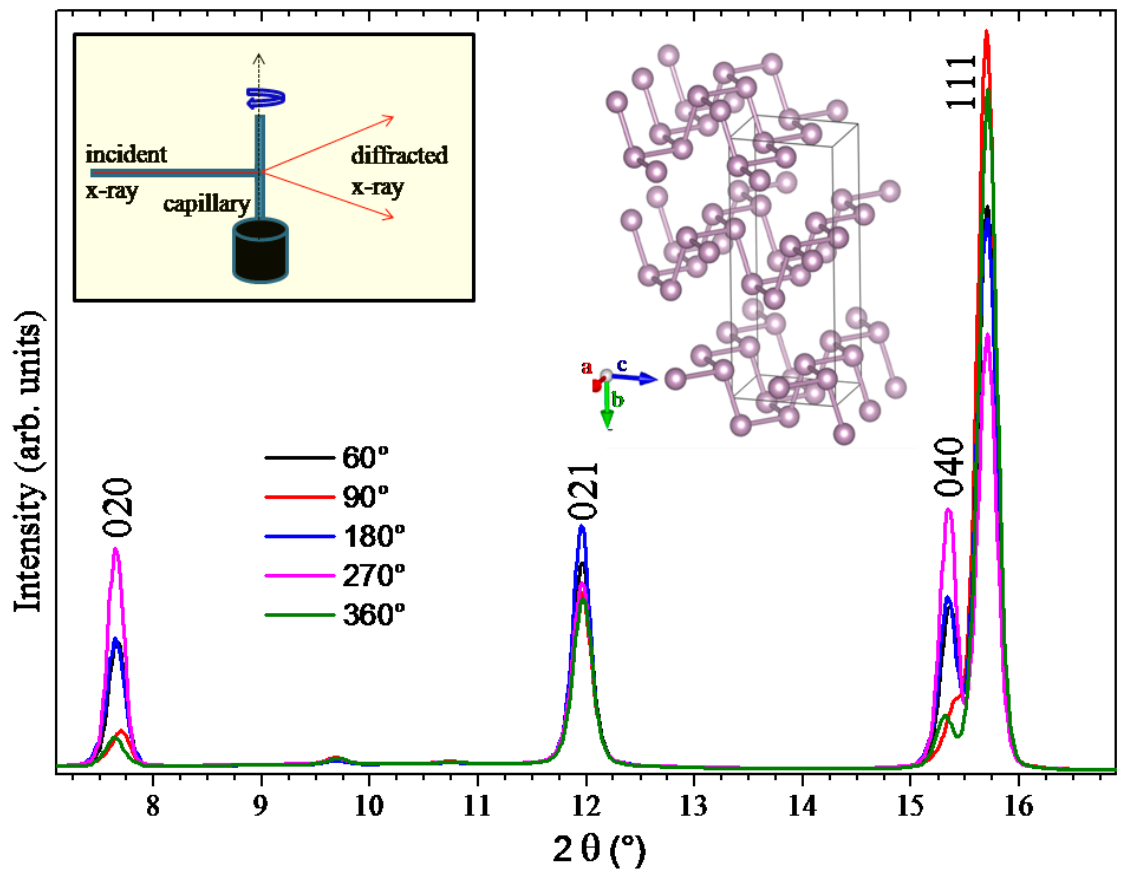

Figure 1. Ambient pressure powder diffraction data from a capillary at a photon wavelength of $0.7 \AA$ with different total amounts of rotation angles (indicated in legends) during the data collection for a fixed exposure time of $10 \mathrm{~s}$. Changing intensities of the peaks area clear indication of the preferred orientation. Left inset shows a schematic of the experimental geometry explaining the rotation angles. Right inset presents the crystal structure of BP.

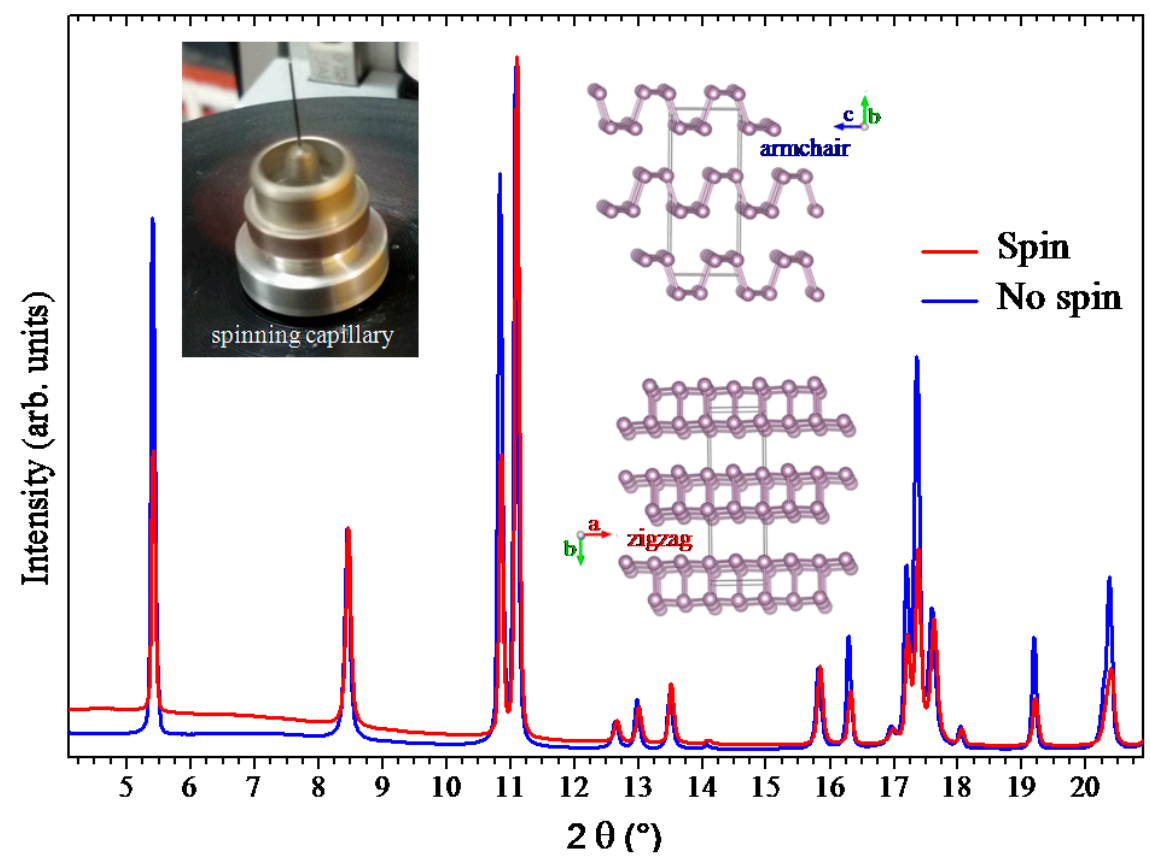

Figure 2. Ambient pressure powder diffraction data from a capillary at a photon wavelength of $0.5007 \AA$ with and without spinning (legends Spin and No-spin). Left inset shows the photo of a spinning capillary. Spinning speeds are above 1000 rotations per minutes. Right insets show crystal structure view along $a$ and $c$. Phosphorus chains are arranged in the armchair manner along $c$ and in a zigzag fashion along $a$. 


\subsection{Rietveld Refinement Results}

In Figure 3, we present the Rietveld refinement results for the BP. Refinements were carried out, including the preferred orientation effects of the $(0 k 0)$ reflections. As expected, the obtained structural parameters were in good agreement with the reported results [20].

In general, for two-dimensional systems, it is a general practice to label the layer plane as the $a-b$ plane and the out-of-plane direction as the $c$ direction. Due to such a tradition, in the literature for the BP system, one can sometimes observe crystal axis directions to be conveniently interchanged to label the out-of-plane direction to be $c$ with the in-plane direction, with the armchair and zigzag P-P atomic arrangement, to be the $a-b$ plane [21]. Another practice is to label the out-of-plane $z$ direction as $z(b)$. However, as is obvious, such nomenclatures do not essentially change the description of the BP system. In Figure 3, we present a zoom over the diffraction data focusing on the three specific $d$-spacings: the (020) peak around $5.26 \AA$, the (200) peak around $1.64 \AA$ and the (002) peak around $2.11 \AA$. The experimental configuration we used permits us to readily analyze the evolution of the peak positions of these characteristic reflections by a simple peak fit. This analysis can readily yield the temperature-dependent changes in the $a, b$, and $c$ lattice parameters. We utilized such an approach to obtain the temperature-dependent lattice parameters' evolution in the temperature range of 85-320 K. We note that there are no structural phase transitions occurring in this temperature range as is evident from the identical kinds of diffraction patterns across the entire temperature range.
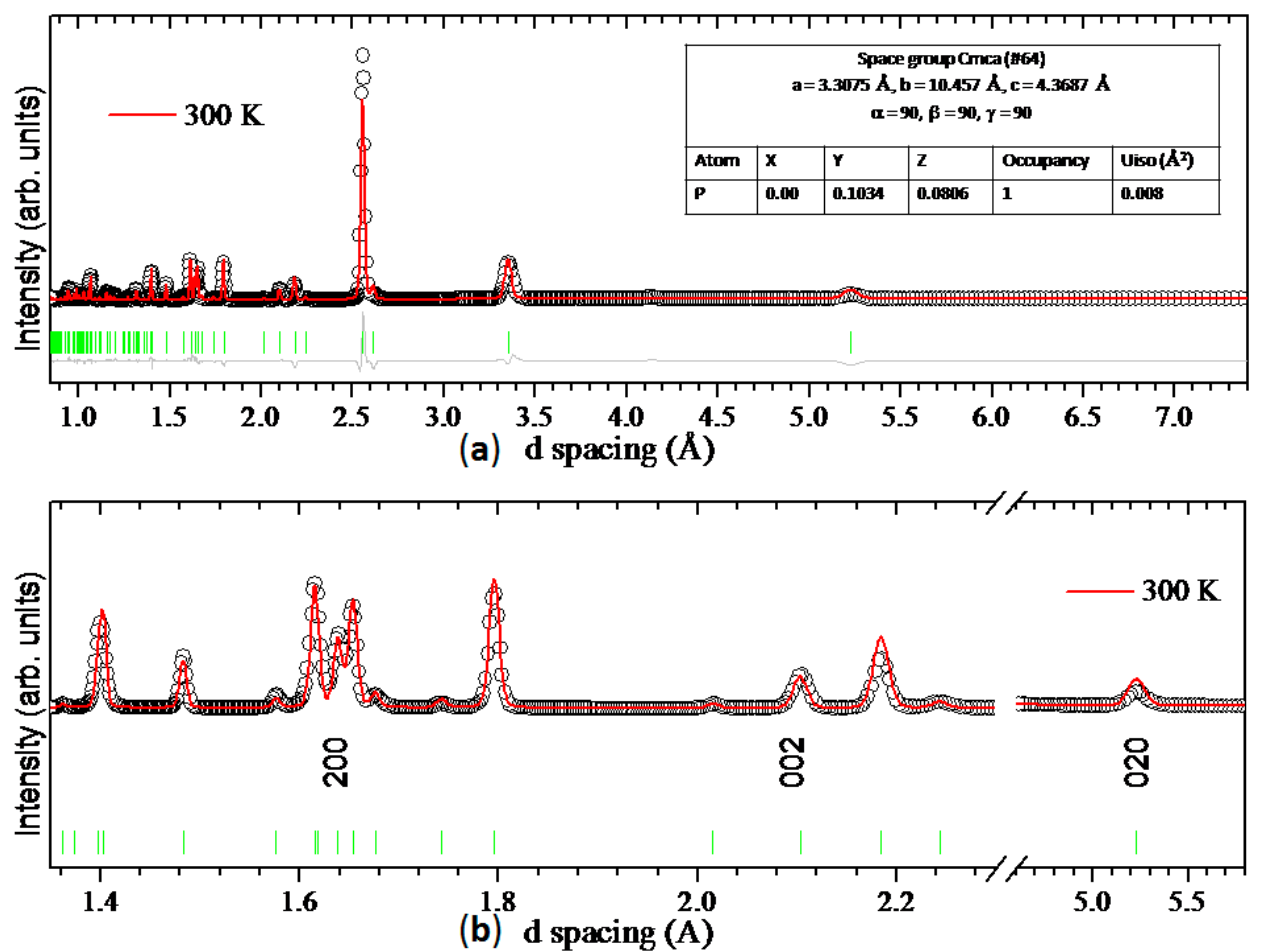

Figure 3. (a) Ambient pressure powder diffraction pattern of black phosphorus together with the Rietveld refinement results at $300 \mathrm{~K}$. (b) A zoom over the d-spacing region between 1.3-2.3 $\AA$ and 4.8-5.8 A showing the (200), (002) and (020) reflections. These data were collected using a wavelength of $0.7 \AA$ in a standard capillary diffraction geometry with a collection time of $30 \mathrm{~s}$ with a continuous 360 degree capillary oscillation normal to the beam direction.

\subsection{Temperature-Dependent Diffraction and Correlation between Structural and Macroscopic Properties of Black Phosphorus}

The $d$-spacings of the (200), (020) and (002) diffraction peaks for the selected four temperatures are shown respectively in Figure 4a-c. Note that the (200) peak (Figure 4a) is sandwiched between 
two other peaks, but clearly independent. A gradual shrinkage of the BP unit-cell with the lowering temperature can be readily observed from the downward shift of these peaks (decreasing $d$-spacing values). Dense sampling in the range of $320 \mathrm{~K}$ to $85 \mathrm{~K}$ (step size $5 \mathrm{~K}$ or smaller) permitted us to systematically follow the temperature-dependent evolution of the lattice parameters. In order to facilitate a comparison of the temperature-dependent changes of the $a, b$, and $c$ lattice parameters together, we considered the relative lattice spacing defined as $a_{T} / a_{320 \mathrm{~K}}, b_{T} / b_{320 \mathrm{~K}}$ and $c_{T} / c_{320 \mathrm{~K}}$ where $a_{T}$ is the $a$ lattice parameter at temperature $\mathrm{T}$ and $a_{320 \mathrm{~K}}$ is the $a$ lattice parameter at $320 \mathrm{~K}$. The relative lattice parameters, $a_{T} / a_{320 \mathrm{~K}}, b_{T} / b_{320 \mathrm{~K}}$ and $c_{T} / c_{320 \mathrm{~K}}$, obtained from such an analysis, are presented in Figure 5a. As expected, all three lattice constants decreased with the temperature; however, the rate of compression was different for the different directions.

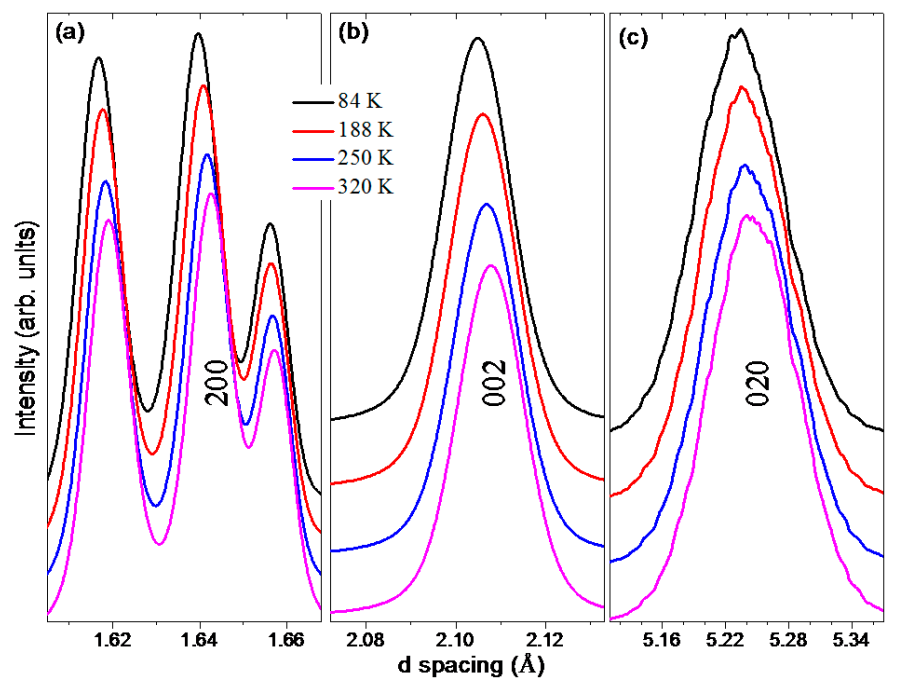

Figure 4. (a-c) A zoom over the $d$-spacing region between 1.3-2.3 $\AA$ and 4.8-5.8 $\AA$ showing the (200), (002) and (020) reflections at several different temperatures. These data were collected using wavelength $0.7 \AA$ in a standard capillary diffraction geometry with a collection time of $30 \mathrm{~s}$ with a continuous 360 degree capillary oscillation normal to the beam direction.
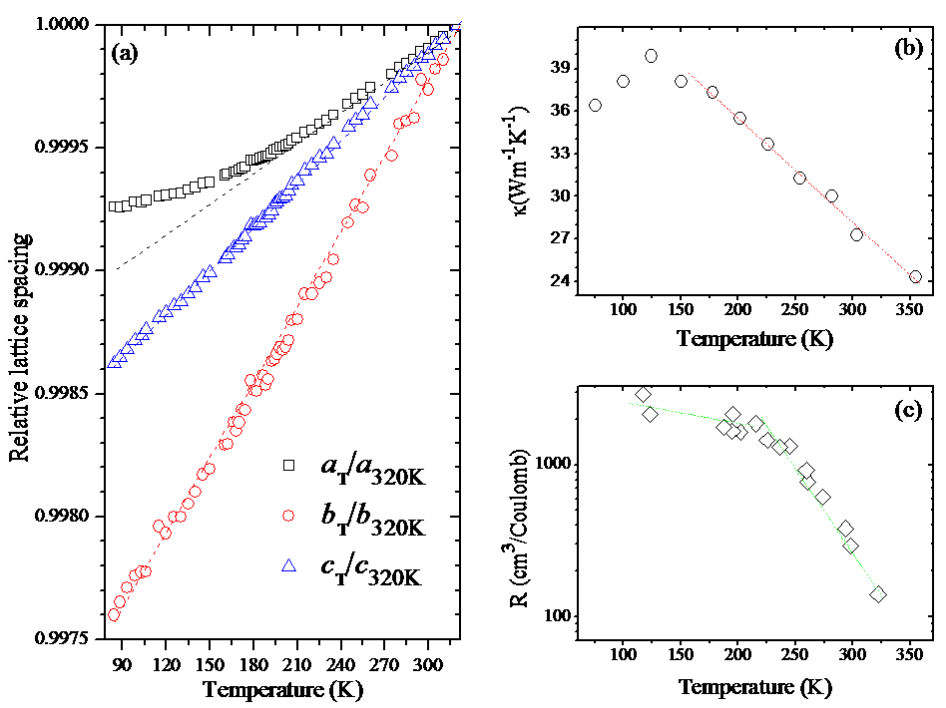

Figure 5. (a) Temperature dependence of the relative lattice spacing of black phosphorus (BP) in the temperature range $85 \mathrm{~K}$ to $320 \mathrm{~K}$. (b) Thermal conductivity ( $\mathrm{K}$ ) vs. temperature of a BP nano-ribbon with a thickness $170 \mathrm{~nm}$ and width $540 \mathrm{~nm}$, data taken from [22]. (c) Temperature dependence of the Hall Coefficient, data taken from [23]. 
As mentioned in the earlier subsection, the intra-layer interaction being the van der Waals type permits a larger compressibility compared to the in-plane lattice parameters. Interestingly, there is also a distinction in the thermal behavior of the in-plane lattice parameters, one of which has a slightly larger compressibility while the other is less compressible. The strong P-P covalent bond in the zigzag direction limits the compressibility along this bond direction. In particular, below $200 \mathrm{~K}$, there is a distinct change in the temperature dependence. Several macroscopic properties of black phosphorus (BP), such as the Hall coefficient, thermal conductivity, etc., show a nonlinear temperature dependence [22,23]. Indeed, interesting temperature-dependent changes in some of these macroscopic properties make BP an ideal candidate for several potential practical applications [24,25]. In Figure $5 b$,c, we present respectively the thermal conductivity $\mathrm{k}\left(\mathrm{Wm}^{-1} \cdot \mathrm{K}^{-1}\right)$ and Hall coefficient $\mathrm{R}$ $\left(\mathrm{cm}^{3} /\right.$ Columb) of BP as functions of the temperature in the range of $85 \mathrm{~K}$ to $320 \mathrm{~K}$. Until $210 \mathrm{~K}$, the Hall coefficient showed a steep increase; however, below $210 \mathrm{~K}$, there was a change in the rate (note the $y$-axis is in logarithmic scale). Recently, the thermal conductivity of BP nanoflakes was careful studied by Lee et al. [23]. The thermal conductivity reported for these nanoflakes also showed a distinct change in this temperature range. In a recent study, Wang et al. [26] suggested that anisotropy in the thermal transport properties of bulk BP might be of particular interest in the fabrication of thermoelectric/optoelectronic devices. Recently, the temperature dependence of the band-gap of BP studied by ab initio calculations shed some light on the anomalous band-gap opening with the increasing volume [27]. The calculated temperature dependence of the lattice parameters along the armchair, zigzag and stacking directions had a good correspondence with the data shown in Figure 5a. Thus, we can conclude that the present results permit us to make a correlation between the structural modulations and macroscopic properties in two-dimensional systems such as BP.

\section{Discussion}

In several cases, the macroscopic properties of materials are closely correlated to the underlining atomic arrangement. Such a relation indeed implies that the fine-tuning of the structural properties has a direct implication on the macroscopic properties. For example, tuning the oxygen ordering with a thermal treatment indeed tunes the superconducting properties of the $\mathrm{La}_{2} \mathrm{CuO}_{4+y}$ cuprate superconductor [2]. An increase in the atomic disorder upon nanostructuring by ball-milling negatively affects the hydrogen sorption properties of $\mathrm{LaNi}_{5}$ [4]. Fine-tuning the stochiometry in a $\mathrm{CePd}_{x} \mathrm{Ge}_{2-x}$ compound provides novel systems with significantly diverse magnetic properties [14]. Nanoparticles of $\operatorname{Pd}_{x} \mathrm{Cu}_{1-x}(x=0,0.25,0.5,0.75$ and 1$)$ are found to have varying catalytic conversion efficiencies for benzylamine to dibenzylimine, with the best results for $x=0.5$ having an ordered body-centered cubic structure unlike $x=0,0.25,0.75$ and 1 which have a face-centered cubic structure [28]. Compared to such direct manipulation of the structural properties, modulation of the structural parameters with temperature, even in the absence of phase transitions, also seems to be in close correlation with the temperature-dependent macroscopic properties such as electrical transport [29]. Such effects are often readily observable, even using bulk structural probes such as diffraction. In two-dimensional systems, such effects can manifest more evidently. In the case of black phosphorus (BP), linear thermal contraction of the out-of-plane axis (stacking direction) is an expected effect considering the fact that the 2- $d$ layers are connected by a weak van der Waals interaction. Temperature can also lead to linear compression along the armchair direction, as it can easily accommodate the stress from the contraction. On the other hand, in the zigzag chain direction, strong P-P covalent bonds are increasingly less susceptible to the decreasing temperature due to the strong covalent nature of this bond. These effects can be readily observed in an amplified way in the pressure studies in the low-pressure regime [30]. Such a microscopic picture can also explain the subtle structural changes of $\mathrm{BP}$ within the temperature range covered in the present study. To conclude, the present results in BP demonstrate a clear correlation between the structural modifications and its macroscopic properties. 


\section{Materials and Methods}

Black phosphorus (BP) as obtained from SigmaAldrich ${ }^{\circledR}$ was used for the present study. Considering the possibility of degradation of BP due to prolonged air exposure, all sample handlings (grinding and transferring to a protective medium, silicone oil) were carried out inside a $\mathrm{N}_{2}$ filled glove box. X-ray diffraction experiments in still and spinning modes were carried out at the newly commissioned dedicated high pressure powder diffraction instrument, the Xpress beamline (Elettra-Sincrotrone Trieste S.C.p.A., Trieste, Italy). During the experiments, Xpress beamline was set to provide intense monochromatic x-ray beam of wavelength $0.5007 \AA$. A custom made pin-hole of diameter around $20 \mu \mathrm{m}$ was used for defining the final beam. A MAR345 image plate detector (marXperts $\mathrm{GmbH}$, Norderstedt, Germany) was used for recording the diffraction pattern. X-ray powder diffraction (XRPD) analyses have been performed at the X-ray diffraction beamline (XRD1) (Elettra-Sincrotrone Trieste S.C.p.A., Trieste, Italy). Temperature dependent measurements were carried out at the XRD1 beamline of Elettra using an Oxford Cryostream 700 series (Oxford Cryosystems Ltd., Oxford, United Kingdom). Data have been collected using a monochromatic wavelength of $0.700 \AA(17.71 \mathrm{keV})$ and $200 \times 200 \mu^{2}$ spot size, using a Dectris Pilatus $2 \mathrm{M}$ hybrid-pixel area detector (DECTRIS Ltd., Baden-Daettwil, Switzerland).. BP powder has been packed in borosilicate capillaries with a $300 \mu \mathrm{m}$ diameter (10 $\mu \mathrm{m}$ wall thickness). Bi-dimensional powder patterns have been integrated using Fit2D program [31] after preliminary calibration of hardware setup, using a capillary filled with $\mathrm{LaB}_{6}$ standard reference powder (NIST 660a). Structural parameters were determined from the diffraction pattern by Rietveld refinements using GSAS package [32].

Acknowledgments: The authors acknowledge the Elettra staff for all their support during measurements. B.J. acknowledges the award of the IISc-ICTP fellowship from IISc Bangalore and ICTP Trieste. He also acknowledges the financial support of Elettra-Sincrotrone Trieste for the participation in the 13th international conference ASCIN 2016 to present the results provided in this manuscript.

Author Contributions: B.J. conceived the experiments; N.D. and B.J. performed the ambient T and T-dependent XRD measurements at the XRD1 beamline. P.L. and B.J. performed the measurements at the Xpress beamline. B.J. analyzed the data and interpreted the results in consultation with others. All authors contributed to the manuscript preparation.

Conflicts of Interest: The authors declare no conflict of interest.

\section{References}

1. Poccia, N.; Fratini, M.; Ricci, A.; Campi, G.; Barba, L.; Vittorini-Orgeas, A.; Bianconi, G.; Aeppli, G.; Bianconi, A. Evolution and control of oxygen order in a cuprate superconductor. Nat. Mater. 2011, 10, 733-736. [CrossRef] [PubMed]

2. Ricci, A.; Poccia, N.; Campi, G.; Coneri, F.; Caporale, A.S.; Innocenti, D.; Burghammer, M.; Zimmermann, M.; Bianconi, A. Multiscale distribution of oxygen puddles in 1/8 doped $\mathrm{YBa}_{2} \mathrm{Cu}_{3} \mathrm{O}_{6.67}$. Sci. Rep. 2013, 3, 2383. [CrossRef] [PubMed]

3. Campi, G.; Bianconi, A.; Poccia, N.; Bianconi, G.; Barba, L.; Arrighetti, G.; Innocenti, D.; Karpinski, J.; Zhigadlo, N.D.; Kazakov, S.M.; et al. Inhomogeneity of charge-density-wave order and quenched disorder in a high-Tc superconductor. Nature 2015, 525, 359-362. [CrossRef] [PubMed]

4. Joseph, B.; Schiavo, B. Effects of ball-milling on the hydrogen sorption properties of LaNi 5 . J. Alloys Compd. 2009, 480, 912-916. [CrossRef]

5. Maugeri, L.; Simonelli, L.; Iadecola, A.; Joseph, B.; Okubo, M.; Honma, I.; Wadati, H.; Mizokawa, T.; Saini, N.L. Temperature dependent local structure of $\mathrm{LiCoO}_{2}$ nanoparticles determined by Co K-edge X-ray absorption fine structure. J. Power Sour. 2013, 229, 272-276. [CrossRef]

6. Joseph, B.; Mohapatra, S.; Lenka, H.P.; Kuiri, P.K.; Mahapatra, D.P. Size saturation in low energy ion beam synthesized Au nanoclusters and their size redistribution with O irradiation. Thin Solid Films 2005, 492, 35-40. [CrossRef]

7. Mathew, S.; Satpati, B.; Joseph, B.; Dev, B.N.; Nirmala, R.; Malik, S.K.; Kesavamoorthy, R. Magnetism in C $60_{0}$ films induced by proton irradiation. Phys. Rev. B 2007, 75, 075426. [CrossRef] 
8. Joseph, B.; Sandeep, C.S.S.; Sekhar, B.R.; Mahapatra, D.P.; Philip, R. Nonlinear optical properties of MeV and keV ion beam synthesized Ag nanoclusters. Nucl. Instrum. Methods Phys. Res. Sect. B 2007, 265, 631-636. [CrossRef]

9. Iadecola, A.; Agrestini, S.; Filippi, M.; Simonelli, L.; Joseph, B.; Mahajan, D.; Saini, N.L. Local structure of $\mathrm{ReFeAsO}(\mathrm{Re}=\mathrm{La}, \mathrm{Pr}, \mathrm{Nd}, \mathrm{Sm})$ oxypnictides studied by Fe K-edge EXAFS. EPL 2009, 87, 26005. [CrossRef]

10. Bendele, M.; Marini, C.; Joseph, B.; Pierantozzi, G.M.; Caporale, A.S.; Bianconi, A.; Pomjakushina, E.; Conder, K.; Krzton-Maziopa, A.; Irifune, T.; et al. Interplay of electronic and lattice degrees of freedom in $A_{1-x} \mathrm{Fe}_{2-y} \mathrm{Se}_{2}$ superconductors under pressure. Phys. Rev. B 2013, 88, 180506. [CrossRef]

11. Malavasi, L.; Artioli, G.A.; Kim, H.; Maroni, B.; Joseph, B.; Ren, Y.; Proffen, T.; Billinge, S.J.L. Local structural investigation of $\mathrm{SmFeAsO}_{1-x} \mathrm{~F}_{x}$ high temperature superconductors. J. Phys. Condens. Matter 2011, 23, 272201. [CrossRef] [PubMed]

12. Xu, W.; Marcelli, A.; Joseph, B.; Iadecola, A.; Chu, W.S.; Gioacchino, D.D.; Bianconi, A.; Wu, Z.Y.; Saini, N.L. Local structural disorder in REFeAsO oxypnictides by RE $\mathrm{L}_{3}$-edge XANES. J. Phys. Condens. Matter 2010, 22, 125701. [CrossRef] [PubMed]

13. Sarkar, S.; Subbarao, U.; Joseph, B.; Peter, S.C. Mixed valence and metamagnetism in a metal flux grown compound $\mathrm{Eu}_{2} \mathrm{Pt}_{3} \mathrm{Si}_{5}$. J. Solid State Chem. 2015, 225, 181-186. [CrossRef]

14. Sarkar, S.; Banerjee, S.; Halappa, P.; Kalsi, D.; Mumbaraddi, D.; Ghara, S.; Pati, S.K.; Sundaresan, A.; da Silva, I.; Rayaprol, S.; et al. Synthetically tuned structural variations in $\mathrm{CePd}_{x} \mathrm{Ge}_{2-x}(x=0.21,0.32,0.69)$ towards diverse physical properties. Inorg. Chem. Front. 2017, 4, 241-255. [CrossRef]

15. Nandi, P.; Giri, C.; Joseph, B.; Rath, S.; Manju, U.; Topwal, D. $\mathrm{CH}_{3} \mathrm{NH}_{3} \mathrm{PbI}_{3}$, a potential solar cell candidate: Structural and spectroscopic investigations. J. Phys. Chem. A 2016, 120, 9732-9739. [CrossRef] [PubMed]

16. Ling, X.; Wang, H.; Huang, S.; Xia, F.; Dresselhaus, M.S. The renaissance of black phosphorus. Proc. Natl. Acad. Sci. USA 2015, 112, 4523-4530. [CrossRef] [PubMed]

17. Castellanos-Gomez, A. Black phosphorus: Narrow gap, wide applications. J. Phys. Chem. Lett. 2015, 6, 4280-4291. [CrossRef] [PubMed]

18. Lee, T.H.; Kim, S.Y.; Jang, H.W. Black phosphorus: Critical review and potential for water splitting photocatalyst. Nanomaterials 2016, 6, 194. [CrossRef]

19. Bridgman, P.W. Two new modifications of phosphorus. J. Am. Chem. Soc. 1914, 36, 1344-1363. [CrossRef]

20. Brown, A.; Rundqvist, S. Refinement of the crystal structure of black phosphorus. Acta. Cryst. 1965, 19, 684-685. [CrossRef]

21. Qiao, J.; Kong, X.; Hu, Z.-X.; Yang, F.; Ji, W. High mobility transport anisotropy and linear dichroism in few-layer black phosphorus. Nat. Comm. 2014, 5, 4475. [CrossRef] [PubMed]

22. Keyes, R.W. The electrical properties of black phosphorus. Phys. Rev. B 1953, 92, 580-584. [CrossRef]

23. Lee, S.; Yang, F.; Suh, J.; Yang, S.; Lee, Y.; Guo, L.; Choe, H.S.; Suslu, A.; Chen, Y.; Ko, C.; et al. Anisotropic in-plane thermal conductivity of black phosphorus nanoribbons at temperatures higher than $100 \mathrm{~K}$. Nat. Comm. 2015, 6, 8573. [CrossRef] [PubMed]

24. Ling, Z.P.; Sakar, S.; Mathew, S.; Zhu, J.-T.; Gopinadhan, K.; Venkatesan, T.; Ang, K.-W. Black phosphorus transistors with near band edge contact Schottky barrier. Sci. Rep. 2015, 5, 18000. [CrossRef] [PubMed]

25. Liu, F.; Zhu, C.; You, L.; Liang, S.-J.; Zheng, S.; Zhou, J.; Fu, Q.; He, Y.; Zeng, Q.; Fan, H.J.; et al. 2D Black Phosphorus $/ \mathrm{SrTiO}_{3}$-Based Programmable Photoconductive Switch. Adv. Mater. 2016, 28, 7768-7773. [CrossRef] [PubMed]

26. Wang, Y.; Xu, G.; Hou, Z.; Yang, B.; Zhang, X.; Liu, E.; Xi, X.; Liu, Z.; Zeng, Z.; Wang, W.; et al. Large anisotropic thermal transport properties observed in bulk single crystal black phosphorus. Appl. Phys. Lett. 2016, 108, 092102. [CrossRef]

27. Villegas, C.E.P.; Rocha, A.R.; Marini, A. Anomalous temperature dependence of the band-gap in black phosphorus. Nano Lett. 2016, 16, 5095-5101. [CrossRef] [PubMed]

28. Marakatti, V.S.; Sarma, S.C.; Joseph, B.; Banerjee, D.; Peter, S.C. Synthetically tuned atomic ordering in $\mathrm{PdCu}$ nanoparticles with enhanced catalytic activity toward solvent-free Benzylamine oxidation. ACS Appl. Mater. Interfaces 2017, 9, 3602-3615. [CrossRef] [PubMed]

29. Joseph, B.; Marini, C.; Demitri, N.; Capitani, F.; Bernasconi, A.; Zhou, W.; Xing, X.; Shi, Z. Temperature dependent structural modulation in $\mathrm{Ca}_{0.82} \mathrm{La}_{0.18} \mathrm{FeAs}_{2}$ pnictide supercondcutors. Supercond. Sci. Technol. 2015, 28, 092001. [CrossRef] 
30. Cartz, L.; Srinivasa, S.R.; Riedner, R.J.; Jorgensen, J.D.; Worlton, T.G. Effect of pressure on bonding in black phosphorus. J. Chem. Phys. 1979, 71, 1718-1721. [CrossRef]

31. Hammersley, A.P.; Svensson, S.O.; Hanfland, M.; Fitch, A.N.; Hausermann, D. Two-dimensional detector software: From real detector to idealized image or two-theta scan. J. High Press. Res. 1996, 14, 235-248. [CrossRef]

32. Larson, A.C.; von Dreele, R.B. General Structure Analysis System (GSAS). Los Alamos Natl. Lab. Rep. 2000, LAUR, 86-748. 